\title{
Spontaneous Variability in Minute Ventilation Oxygen Consumption and Heart Rate of Low Birth Weight Infants
}

\author{
KARL SCHULZE, ${ }^{(30)}$ RAM KAIRAM, MARK STEFANSKI, ROBERT SCIACCA, DAVID BATEMAN, \\ RALPH DELL, AND L. S. JAMES \\ Departments of Pediatrics and Medicine, College of Physicians and Surgeons, Columbia University, \\ New York, New York, USA
}

\begin{abstract}
Summary
Continuous measurements of minute ventilation $\left(\dot{V}_{\mathrm{I}}\right)$, oxygen consumption $\left(\dot{\mathrm{V}}_{\mathrm{O}_{2}}\right)$, heart rate $(\mathrm{HR})$, activity, and temperature were made in eleven low birth weight infants during the interval between feedings. Significant increases in $\dot{V}_{\mathrm{I}}, \dot{\mathrm{V}}_{\mathrm{O}_{2}}$, and HR were noted between quiet and active sleep. $\left(\dot{V}_{\text {I Active }}-\dot{V}_{I}\right.$ Quiet $\left./ \dot{V}_{\text {I Quiet }}\right)$ $\times 100=18.4 \% \dot{\mathrm{V}}_{\mathrm{O}_{2} \text { Active }}-\dot{\mathrm{V}}_{\mathrm{O}_{2} \text { Quiet }} / \dot{\mathrm{V}}_{\mathrm{O}_{2} \text { Quiet }} \times 100=10.1 \%$ and $\left.H R_{\text {Active }}-H R_{\text {Quiet }} / H_{R_{\text {Quiet }}}\right) \times \mathbf{1 0 0}=\mathbf{6 . 4 \%}$. Significant differences were also noted within epochs of the same state of sleep: mean slope $\dot{\mathrm{V}}_{\mathrm{I}}$ versus time in epoch $(\mathrm{t})=-156 \mathrm{ml} / \mathrm{kg} \cdot \mathrm{min} / \mathrm{hr}, \dot{\mathrm{V}}_{\mathrm{O}_{2}}$ versus $\mathrm{t} .=1.49 \mathrm{ml} / \mathrm{kg} \bullet \mathrm{min} / \mathrm{hr}$ and $\mathrm{HR}$ versus $\mathrm{t}=-15.0$ beats $/ \mathrm{min} / \mathrm{hr}$. Differences between successive epochs of the same state of sleep were also observed: $\dot{V}_{\mathrm{I}},+5.9$ to $46.6 \%$; $\dot{\mathrm{V}}_{\mathrm{O}_{2}}, 4.7$ to $24.6 \%$; $\mathrm{HR}, 1.0$ to $9.7 \%$. These differences were related to the length of time after feeding. These data indicate that steady state conditions do not occur in growing low birth weight infants and that the design of studies of respiration and metabolism in these infants should include continuous assessment of the state of sleep or activity and time after feeding to ensure that experimental and control periods are truly comparable.
\end{abstract}

\section{Speculation}

The significant variability in minute ventilation, oxygen consumption, and heart rate which occurs spontaneously in low birth weight infants secondary to changes in activity and postprandial interval must be acknowledged in the design of clinical research. It is probable that these factors influence not only mean levels of metabolic and respiratory activity but also the sensitivity to experimental stimuli such as hypoxia, hypercarbia, and drugs.

The oxygen consumption and carbon dioxide production of two low birth weight infants were first measured by Hasselbalch in 1904, utilizing open-circuit, indirect calorimetry similar in principle to techniques in use today (11). In the original report, it was observed that basal metabolism is influenced by activity, feeding, birth weight, and postnatal age. It was also suggested that temperature should be investigated for possible effects on metabolism. Thus, this initial clinical study of energy exchange in the low birth weight infant identified or alluded to most of the major factors which contribute to the variability in this fundamental physiologic measurement.

Researchers have refined both the theory and the methodology for gaseous metabolism and now recognize that activity, including different states of sleep (25), the amount and quality of dietary intake (15), and the thermal environment $(6,7)$ have important influences on metabolism and related respiratory and circulatory functions. The metabolic effects of activity, feeding, and temperature must be eliminated if true basal conditions are to be achieved. However, in practice this is not possible, and true basal metabolism of low birth weight infants has never been measured.

Recognizing these limitations, investigators have attempted to control the effects of activity, feeding, and temperature through careful definition of thermal conditions, time in the feeding cycle, and the states of sleep and wakefulness of the infant, thereby establishing physiologic conditions which have been regarded as "steady state" for the duration of the study period. That steadystate conditions were actually produced by the control measures has not been documented, and the spontaneous physiologic variability experienced by the low birth weight infant over time has not been defined.

The purpose of this study, then, was to quantitate effects of the state of activity and the prandial cycle on minute ventilation $\left(\dot{V}_{1}\right)$, oxygen consumption $\left(\mathrm{V}_{\mathrm{O}_{2}}\right)$, and heart rate (HR) of low birth weight infants. The aim was not only to make mean estimates of $\dot{\mathrm{V}}_{\mathrm{I}}, \dot{\mathrm{V}}_{\mathrm{O}}$, and HR but also to estimate the variability in these measurements and to identify the major contributor to this variability. To accomplish these objectives, we made continuous minute by minute recordings of $\dot{\mathrm{V}}_{\mathrm{I}}, \dot{\mathrm{V}}_{\mathrm{O}}$, carbon dioxide production, $H R$, electroencephalogram and electroculogram, clinical activity, and body and environmental temperatures of growing low birth weight infants being cared for under standard nursing conditions during most of the postprandial period ( 2 to $3 \mathrm{hr}$ ).

\section{MATERIALS AND METHODS}

The study protocol was reviewed and approved by the Institutional Review Board. Growing low birth weight infants without cardiorespiratory disease were selected for inclusion in the study using parental informed consent as the only entrance criterion. Characteristics of the study population are given in Table 1.

\section{$\dot{\mathrm{V}}_{1}$}

Bias flow pneumotachometry was used for measurement of $\dot{V}_{1}$ utilizing principles defined by Rigatto and Brady (18). A face mask (Bennet No. 5284; Bennett Respiratory Products, Los Angeles, CA) was attached to the face of the infant using a rubber harness designed in our laboratory. A stable measured gas flow was drawn past the face mask at rates of approximately $3500 \mathrm{ml} /$ min. The inspiratory and expiratory flows of the infant were subtracted from and added to the bias flow. Tidal gas flow changes were measured with either a Fleisch 00 pneumotachograph or a screen pneumotachograph attached to a differential pressure transducer (Statham PM 97) which had been zeroed electrically to the bias flow. Integrated inspiratory flow signals were summed continuously each minute and recorded as an increasing analog signal which was automatically reset each minute. The minute volume signal was calibrated by withdrawing standard volumes in a syringe at the point of attachment to the infant. 
Table 1. Characteristics of study infants

\begin{tabular}{|c|c|c|c|c|c|}
\hline Patient & $\begin{array}{l}\text { Birth wt } \\
\text { (g) }\end{array}$ & $\begin{array}{l}\text { Wt at study } \\
\quad(\mathrm{g})\end{array}$ & $\begin{array}{l}\text { Gestational age at birth } \\
\text { (wk) }\end{array}$ & $\begin{array}{l}\text { Postconceptional age at study } \\
\text { (wk) }\end{array}$ & $\begin{array}{l}\text { Length of study } \\
\qquad(\mathrm{min})\end{array}$ \\
\hline 1 & 1100 & 1130 & 32 & 33 & 97 \\
\hline 2 & 980 & 920 & 30 & 31.5 & 110 \\
\hline 3 & 1490 & 1400 & 32 & 33 & 127 \\
\hline 4 & 1220 & 1310 & 31 & 32 & 114 \\
\hline 5 & 950 & 1120 & 28 & 33 & 102 \\
\hline 6 & 1200 & 1390 & 30 & 35 & 146 \\
\hline 7 & 1380 & 1380 & 30 & 32 & 104 \\
\hline 8 & 810 & 890 & 26 & 30 & 127 \\
\hline 9 & 1160 & 1670 & 30 & 37 & 129 \\
\hline 10 & 2180 & 2180 & 34 & 38 & 138 \\
\hline 11 & 650 & 2300 & 27 & 41 & 191 \\
\hline
\end{tabular}

\section{$\dot{\mathrm{V}}_{\mathrm{O}}$}

$\dot{\mathrm{V}}_{\mathrm{O}_{2}}$ was measured by open circuit indirect calorimetry. The exchange of tidal gas produced a decrease in the oxygen concentration of gas downstream from the infant. This small difference was measured directly by a Servomex OA 184 (Servomex Ltd., Sussex, England) differential oxygen analyzer which continuously measured the $\mathrm{FiO}_{2}$ upstream and downstream from the study subject and provided a difference signal. Gas flow rate was measured with a linear mass flow meter. Oxygen uptake was then calculated as the product of gas flow and differential oxygen concentration. Corrections for changes in respiratory exchange were made with a formula derived by Lister et al. (14).

Irregularities in respiratory cycles produced second-to-second variability in alveolar ventilation and secondary variability in the differential oxygen signal. It was necessary therefore to integrate the oxygen signal each minute to obtain an average difference in oxygen concentration for that minute. This was done on line with a voltage to frequency converter and an analog totalizer (North Hills Electronics, Glen Cove, NY). The analog totalizer was reset each minute in synchrony with the other signals.

\section{CARBON DIOXIDE PRODUCTION}

Carbon dioxide production was measured by standard open circuit technique using a Beckman LB-2 infrared carbon dioxide analyzer (Beckman Instruments, Anaheim, CA). The output signal from the analyzer was processed like the oxygen signal yielding minute-to-minute measurements of carbon dioxide production. A complete description of this methodology has been published (21).

\section{$H R$}

The pulse signal was obtained from a HR module (Hewlett Packard $7820 \mathrm{~A}$ ) and linked directly to an analog totalizer. An increment in an analog signal accompanied each QRS complex until the signal was reset each minute. HR totals were then read each minute from the strip chart recorder.

\section{CLINICAL ACTIVITY}

Clinical observations were made continuously by one of the investigators. Each minute, an activity score was assigned for the predominant clinical behavior pattern according to a cording system developed in our laboratory. This system differs from other scoring systems for preterm infants $(6,17)$ in that rapid eye movements are included and respiratory pattern is excluded as criteria for designation of a clinical activity score:

Quiet sleep

1. Flaccid "rag doll" appearance. Body movements limited to startles (sudden contraction of many muscles lasting 1 or 2 sec with an immediate return to a relaxed posture). Rhythmic jaw jerks lasting 1 or 2 sec are also seen.

2. Quiescent with apparent increased tone in the limbs. The increased tone is reflected in the antigravity posturing of extremities. Motor activity is limited to jaw jerks or startles. Active Sleep

3. Small body movements present. Motor activity is more generalized and includes slow intermittent writhing movements, rapid jerky startles, small movements of an extremity or parts thereof, frowns, smiles, chewing and sucking movements. grimaces and other facial activity, grunts, and occasional whimpers.

4. Rapid eye movement observed clinically. Movements are limited to those seen in states 1, 2 and 3. Eyes may occasionally open and close.

Wakefulness

5. Continuous gross body movements. Apparent nonreflex movements of limbs, stretching, writhing, and prolonged startles. Facial activity is usually present. No crying is observed. Eyes may be open or closed, and infant may appear alert.

6. Crying. The features of state 5 are also observed.

7. Recovering period after crying. Regular deep rapid respirations are prominent with little extremity movement. Eyes are usually closed.

\section{NEUROPHYSIOLOGIC ACTIVITY}

Electroencephalogram (EEG), electroculogram, electrocardiogram, and impedance pneumogram were recorded on an Offner type $T$ polygraph. Each minute of the EEG recording was coded according to the predominant pattern of the interval using the characteristic EEG patterns of preterm infant sleep described by Dreyfus-Brisac (9), Parmelee et al. (16), and Anders et al. (1) as listed below:

Quiet Sleep

1. Tracé alternant pattern: bursts of slow waves $(0.5$ to $3 \mathrm{~Hz}) 50$ to $100 \mu \mathrm{V}$ with occasional superimposition of rapid low voltage waves. These bursts, lasting 3 to $8 \mathrm{sec}$, are separated by 4 to $8 \mathrm{sec}$ of low voltage or mixed activity. Tracé discontinu pattern: bursts of high voltage (greater than $100 \mu \mathrm{V}$ ) of 3 to $10 \mathrm{sec}$ duration, separated by periods of attenuated activity lasting 10 to $40 \mathrm{sec}$, rarely $60 \mathrm{sec}$.

2. High voltage, slow wave pattern. Continuous medium-tohigh voltage activity (mostly $75 \mu \mathrm{V}$, occasionally 100 to 150 $\mu \mathrm{V}$ ) with frequencies of 0.5 to $4 \mathrm{~Hz}$. The slow waves are often rhythmic.

Active Sleep

3. Mixed pattern. Predominantly continuous polymorphic activity of 4 to $7 \mathrm{~Hz}$ averaging $50 \mu \mathrm{V}$ in amplitude intermingled with slower waves ( 2 to $4 \mathrm{~Hz}$ ) of slightly higher voltages.

4. Immature rhythmic slowing pattern. Monomorphic high amplitude delta waves of 0.3 to $2.0 \mathrm{~Hz}$ which often occur in extended sequences. They are particularly prominent in the temporal and occipital areas and are often associated with superimposed 10 to $20 \mathrm{~Hz}$ activity ("brushes"). Other activity includes moderate voltage 2 to $8 \mathrm{~Hz}$ wave forms.

5. Low voltage irregular pattern. Continuous 4 to $7 \mathrm{~Hz}$ activity, 
sometimes rhythmic, with voltages predominantly 20 to 30 $\mu \mathrm{V}$.

Wakefulness

6. Artifact. Records of poor quality and uninterpretable due to base line shifts from movement, crying, sweating, or handling. Excessive electromyogram artifact obscuring nature of frequency.

\section{ADDITIONAL OBSERVATIONS}

Temperature was recorded every minute from thermistors in the environment above the baby from a peripheral skin site with a Yellow Springs telethermometer YSI 47 (Yellow Springs Instruments, Yellow Springs, OH).

All signals were recorded on Soltec KA strip chart recorders (Soltec Inc., Sun Valley, CA) or on the Offner polygraph (Beckman Instruments, Anaheim, CA), and digital values for each minute of the study were obtained by inspection of the paper tracing.

\section{STUDY PROTOCOL}

Immediately before a scheduled feed, the EEG and electroculogram electrodes were placed on the infant and secured with a turban dressing. The infant was then fed, attended, and taken in the isolette to a special diagnostic unit which contains a complete nursing site identical to those in the adjacent intensive care unit.

The mask was attached to the face of the infant and adjusted to allow inspection of the eyes. All instrumentation was precalibrated and functioning at the time the infant was connected to the gas stream. Studies began approximately $30 \mathrm{~min}$ after the feeding was completed. The beginning of the study and other important clinical events were noted with a synchronized event marker and timer on all recording equipment. Throughout the study, the infant remained in the single-walled incubator servocontrolled to a skin temperature of $36.5^{\circ} \mathrm{C}$. Observations were continued until prolonged wakefulness occurred or until preparations for the next feeding were begun.

\section{DATA ANALYSIS}

Digital data for each minute were obtained for $\dot{\mathrm{V}}_{\mathrm{O}_{2}}, \dot{\mathrm{V}}_{\mathrm{I}}, \mathrm{HR}$, temperature (environment, abdomen, and peripheral skin), and neurophysiologic and clinical activity. The data were entered into an IBM 360 computer for storage and analysis. Time series analysis was used to align the observations in time and correct for biologic and methodologic lags.

Data were analyzed in sequential 3-min averages. This average was performed to smooth out random noise in the measurement of oxygen consumption as a result of alterations in oxygen stores occurring in response to short-term variations in alveolar ventilation (10). For analysis, designations of the infant's state for a 3min interval was made according to three separate schemes. In the first analysis, the clinical scores (see clinical scale above) were added for each minute in the 3-min period, and state was designated according to the total as follows: quiet sleep, 3 to 6; active sleep, 7 to 13 ; wakefulness, $\geq 14$. A second, independent analysis was made by summing three consecutive minute neurophysiologic scores (see neurophysiologic scale above) and activity state assigned according to the following scale: quiet sleep, 3 to 6 ; active sleep, 7 to 15 ; wakefulness, $\geq 16$. Clinical and neurophysiologic scores were then summed for each interval and the state of activity was designated as follows: quiet sleep 6 to 12; active sleep, 13 to 29 , and wakefulness, $\geq 30$. A comparison was then made of those intervals having the same clinical and neurophysiologic score throughout the $3 \mathrm{~min}$ with the total data including intervals where clinical and EEG scores disagreed and/or intervals of mixed state (e.g., $2 \mathrm{~min}$ of quiet sleep and $1 \mathrm{~min}$ of active sleep). All results were similar regardless of the method of analysis. Data presented are from the analysis of combined scores and include all the data recorded during each study.

\section{RESULTS}

There was a decrease in $\dot{\mathrm{V}}_{\mathrm{I}}, \dot{\mathrm{V}}_{\mathrm{O}_{2}}$, and $\mathrm{HR}$ as the infant went from wakefulness to active sleep to quiet sleep. A typical record of minute-to-minute measurement of $\dot{\mathrm{V}}_{\mathrm{I}}, \dot{\mathrm{V}}_{\mathrm{O}_{2}}$, and HR in growing low birth weight infant (infant 1) after a feeding is shown in Figure 1. Mean and standard deviation for all variables during quiet sleep, active sleep, and wakeful states in the same infant are shown in Figure 2 . All variables increased with increased activity. Mean and standard deviations for $\dot{\mathrm{V}}_{1}, \dot{\mathrm{V}}_{\mathrm{O}_{2}}$, and HR during three states of activity are presented in Table 2 for the entire study population. All patients except infant 3 showed a higher mean $\overrightarrow{\mathrm{V}}_{\mathrm{O}_{2}}$ in active sleep than in quiet sleep, and all patients showed a higher mean $\dot{V}_{1}$ and $H R$ in active sleep. For all patients, mean percent increases from quiet to active sleep were $\left(\dot{\mathrm{V}}_{\mathrm{I}}\right.$ Active $-\dot{\mathrm{V}}_{\mathrm{I}}$

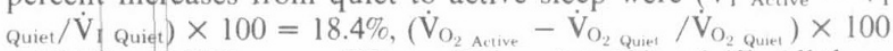
$=10.1 \%$ and $\left(\mathrm{HR}_{\text {Active }}-\mathrm{HR}_{\text {Quiet }} / \mathrm{HR}_{\text {Quiet }}\right) \times 100=6.4 \%$. All three increases were statistically significant $(P<0.01)$, indicating that activity state has a significant effect on $\dot{\mathrm{V}}_{\mathrm{I}}, \dot{\mathrm{V}}_{\mathrm{O}_{2}}$, and HR.

The prandial cycle was also found to influence $\dot{\mathrm{V}}_{\mathrm{I}}, \dot{\mathrm{V}}_{\mathrm{O}_{2}}$, and HR. All discrete epochs of quiet and active sleep lasting longer than $15 \min (N=20)$ were analyzed for trends in each measurement within an epoch of the same state of sleep. The slopes of the regression lines of $\dot{\mathrm{V}}_{\mathrm{I}}, \dot{\mathrm{V}}_{\mathrm{O}_{2}}$, and $\mathrm{HR}$ against time within an epoch of the same state of sleep were calculated. The results are shown in Table 3. The slopes were, with rare exceptions, negative and significantly different from zero $(P<0.01)$. Furthermore, the slopes of the regression lines became increasingly negative as time after feeding increased $\left(\dot{\mathrm{V}}_{\mathrm{I}}, P<0.10 ; \dot{\mathrm{V}}_{\mathrm{O}_{2}}, P<0.05 ; \mathrm{HR}, P<\right.$ $0.05)$. These data indicate that in growing low birth weight infants $\dot{\mathrm{V}}_{\mathrm{I}}, \dot{\mathrm{V}}_{\mathrm{O}_{2}}$, and HR decrease continuously during an epoch of the same state of sleep and that this decrease is greater as the time after feeding increases.

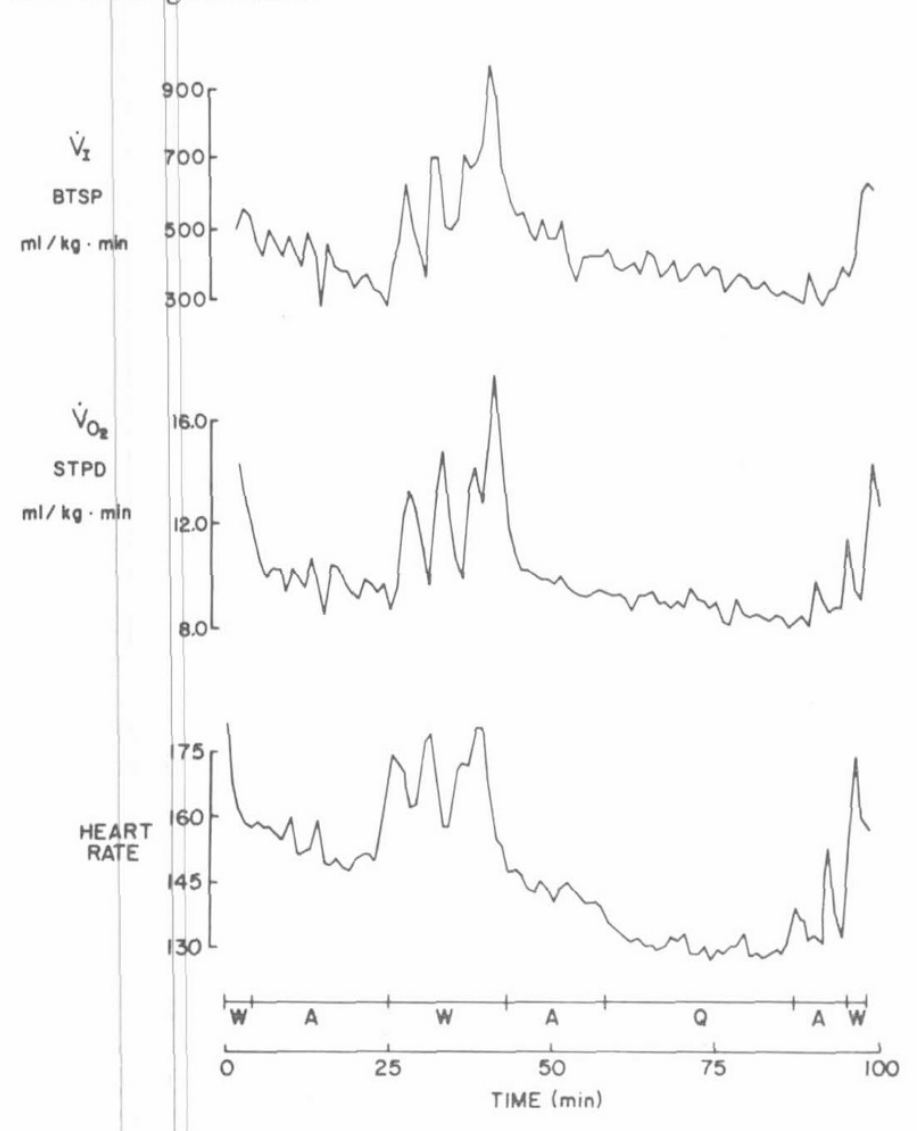

Fig. 1. Continuous minute by minute record of $\dot{\mathrm{V}}_{1}, \dot{\mathrm{V}}_{\mathrm{O}_{2}}$, and $\mathrm{HR}$ of a growing $1130 \mathrm{~g}$ infant. The state of activity is designated above the time scale: $Q$, quiet sleep; $A$, active sleep; $W$, wakefulness. The data are plotted without correction for lag times. Hence, changes in heart rate and ventilation precede associated changes in $\dot{\mathrm{V}}_{\mathrm{O}_{2}}$. 

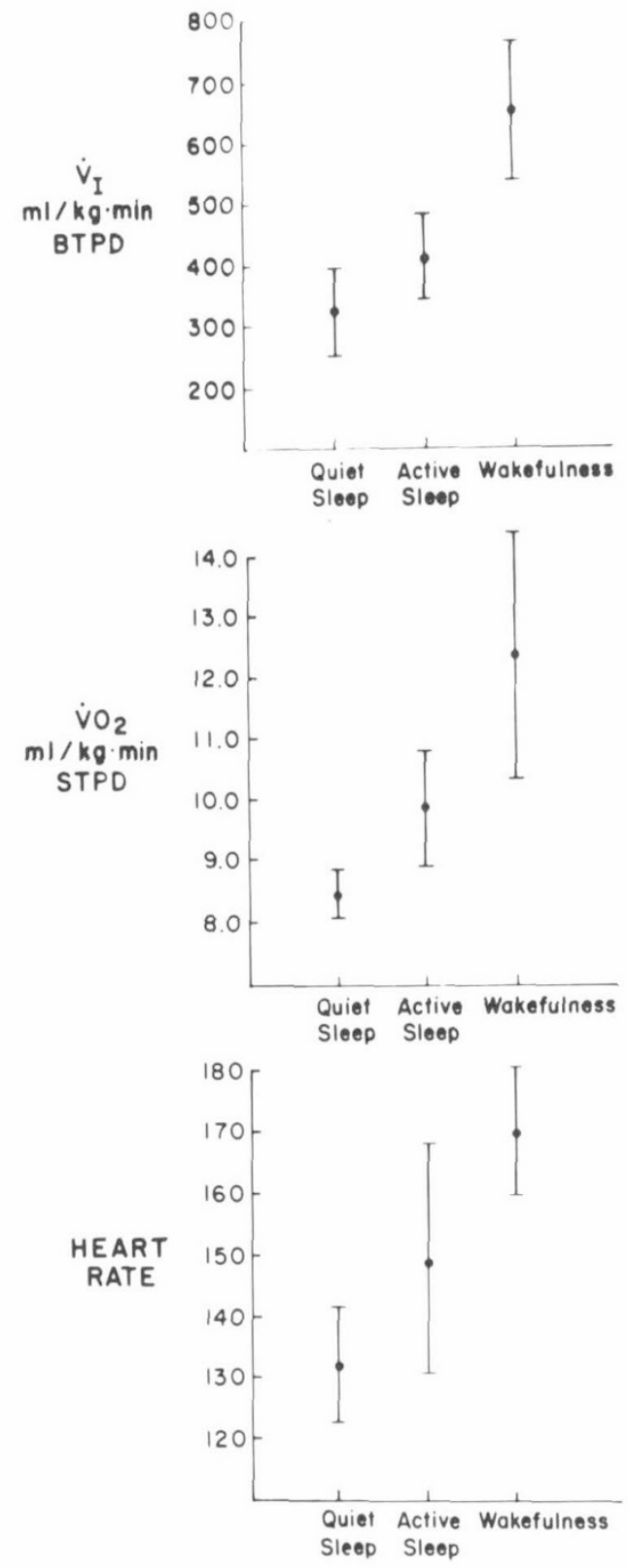

Fig. 2. Mean and standard deviations of 3 min averaged measurements of $\dot{\mathrm{V}}_{1}, \dot{\mathrm{V}}_{\mathrm{O}_{2}}$, and HR during quiet sleep, active sleep, and wakefulness.

Interepoch variability was also found when multiple epochs of active and quiet sleep were examined. Mean values for all variables recorded during the first epoch of active and quiet sleep after feeding were compared to the mean values for a subsequent epoch of the same state of sleep in the same infant. The results are presented in Table 4. Metabolic and respiratory activity was less in the second epoch in all infants.

\section{DISCUSSION}

The objective of this study was to quantitate the effect of sleep state and time after feeding on respiration and metabolism by measuring $\dot{\mathrm{V}}_{\mathrm{I}}, \dot{\mathrm{V}}_{\mathrm{O}_{2}}$, and $\mathrm{HR}$ in low birth weight infants under steady-state conditions. Unlike earlier studies, we utilized continuous measurements of $\dot{\mathrm{V}}_{\mathrm{I}}, \dot{\mathrm{V}}_{\mathrm{O}_{2}}$, and $\mathrm{HR}$ and both neurophysiologic and clinical assessment of state of activity for intervals of several hours. The results shown in Table 2 indicate that statistically significant decreases in $\dot{\mathrm{V}}_{\mathrm{I}}, \dot{\mathrm{V}}_{\mathrm{O}_{2}}$, and $\mathrm{HR}$ do occur with spontaneous decreases in activity of low birth weight infants. In addition, as shown in Tables 3 and $4, \dot{\mathrm{V}}_{\mathrm{I}}, \dot{\mathrm{V}}_{\mathrm{O}_{2}}$, and HR decrease during each sleep epoch as well as between successive episodes of same state of sleep, suggesting the influence of the time in the prandial cycle at which measurements are made.

The mean change in $\dot{V}_{1}$ between quiet and active sleep of $18.4 \%$ which we have observed is similar to the changes of $14 \%$ reported in term infants (3) and of $15.0 \%$ in preterm infants (19). The $10.1 \%$ difference in $\dot{\mathrm{V}}_{\mathrm{O}_{2}}$ consumption between quiet and active sleep is somewhat higher than the $4.8 \%$ difference observed in term infants (25). Our results are contrary to the results of another study which found no difference in $\dot{\mathrm{V}}_{\mathrm{O}_{2}}$ between quiet and active sleep (24). The latter study used clinical criteria only to assess the state of sleep. Our results also support data obtained by early investigators who reported increases in $\dot{\mathrm{V}}_{\mathrm{O}_{2}}$ in preterm infants between epochs of quiet and active sleep defined only by clinical criteria (22). One study reported HR of preterm infants to be higher in active than in quiet sleep (27). However, this finding was not confirmed in a recent study with continuous computer analysis (23). Our results clearly indicate a lower mean HR is associated with quiet sleep.

Downward trends in $\dot{\mathrm{V}}_{\mathrm{O}_{2}}$ within epochs of the same state of sleep have been reported (25), and it has also been noted that there is a decrease in respiratory frequency as the time within an epoch of quiet sleep increased (8). A downward trend has also been reported among successive postprandial epochs of the same state of sleep in respiratory rate and in heart rate $(2,8)$.

Current research strategies are based on the beliefs that epochs of the same activity state in the same infant are physiologically comparable if no major change in nursing conditions has occurred and that a relatively brief measurement of a physiologic variable such as $\dot{\mathrm{V}}_{\mathrm{I}}$ or $\dot{\mathrm{V}}_{\mathrm{O}}$, is representative of the entire epoch under study. In stimulus-response experimentation such as $\mathrm{CO}_{2}$ rebreathing, drug administration, etc., it is then further assumed that differences in control and experimental observations made of the same infant in the same state of activity are related to the experimental stimulus applied during the investigation. This approach is unsound inasmuch as our data indicate that significant differences in $\dot{\mathrm{V}}_{\mathrm{I}}, \dot{\mathrm{V}}_{\mathrm{O}_{2}}$, and HR can occur within the same state of sleep. Further, the differences we have observed are of similar or greater magnitude than many observed experimental outcomes

The physiologic explanation for the tendency for $\dot{V}_{\mathrm{I}}, \dot{\mathrm{V}}_{\mathrm{O}_{2}}$, and HR to decrease continuously after a feeding is not fully understood. It is clear that the feeding process itself may significantly influence metabolism and respiration during the entire postfeeding interval independent of the state of activity. Growing low birth weight infants are usually fed every 3 or $4 \mathrm{hr}$. Feeding provides a number of tactile, thermal, mechanical, and biochemical stimuli which can influence the physiology of the infant to a variable degree and for a variable period of time after completion of the feeding. Stimulation of somatic sensory and vagal neurons is known to alter respiratory function both acutely and for an interval of time after removal of the stimulus $(12,28)$, but it is not thought that the response to tactile stimuli persists into the second and third postprandial hr. Thermal stress is a potent stimulant of metabolism in low birth weight infants and may occur even in single-walled incubators servocontrolled to maintain the abdominal skin temperature of the infant at $36.5^{\circ} \mathrm{C}$ (26). However, no correlation between temperature and metabolic rate was found in our infants. Temporary mechanical distention of the stomach from feeding could cause secondary changes in pulmonary mechanics and account for the course of the observed decreases in all variables after the feeding. Several studies of pulmonary mechanics in low birth weight infants after feedings have not shown unfavorable changes in the postabsorptive period $(13,29)$.

The decrease in metabolism and ventilation during the postprandial interval could also be explained by a gradual adaptation of the infant to the experimental system. Measurements in our laboratory and elsewhere of respiratory frequency, $\dot{\mathrm{V}}_{\mathrm{O}_{2}}$, and HR using standard open-circuit, indirect calorimetry without a face mask have shown similar mean values and the same decreasing trend in all measurements after feeding $(2,5,8)$. No systematic differences in the distribution of state of sleep were noted between studies performed with and without a face mask. On the basis of 
Table 2. $\dot{V}_{I}, \dot{V}_{O_{2}}$, and $H R$ during quiet sleep, active sleep, and wakefulness

\begin{tabular}{|c|c|c|c|c|c|c|c|}
\hline Patient & State & $\begin{array}{c}\dot{\mathrm{V}}_{\mathrm{l}} \\
(\mathrm{ml} / \mathrm{kg}-\mathrm{min} \\
\text { BTPD) }\end{array}$ & $\begin{array}{l}\text { \% Change } \\
\frac{\mathrm{V}_{1} \mathrm{~A}^{1}-\mathrm{V}_{\mathrm{I}} \mathrm{Q}}{\mathrm{V}_{\mathrm{I}} \mathrm{Q}} \times 100\end{array}$ & $\begin{array}{c}\dot{\mathrm{V}}_{\mathrm{O}_{2}} \\
(\mathrm{ml} / \mathrm{kg}-\mathrm{min} \\
\mathrm{STPD})\end{array}$ & $\begin{array}{l}\text { of Change } \\
\frac{\dot{\mathrm{V}}_{\mathrm{O}_{2}} \mathrm{~A}-\dot{\mathrm{V}}_{\mathrm{O}_{2}} \mathrm{Q}}{\mathrm{V}_{\mathrm{O}_{2}} \mathrm{Q}} \times 100\end{array}$ & HR & $\begin{array}{l}\text { \% Change } \\
\frac{H R_{A}-H R_{Q}}{H R_{Q}} \times 100\end{array}$ \\
\hline 1 & $\begin{array}{l}\mathrm{Q} \\
\mathrm{A} \\
\mathrm{W}\end{array}$ & $\begin{array}{l}368 \pm 72^{2} \\
455 \pm 70 \\
699 \pm 118\end{array}$ & 23.6 & $\begin{array}{r}8.47 \pm 0.40 \\
9.86 \pm 0.93 \\
12.39 \pm 2.02\end{array}$ & 16.4 & $\begin{array}{l}133.7 \pm 4.7 \\
148.5 \pm 8.9 \\
169.3 \pm 5.3\end{array}$ & 11.1 \\
\hline 2 & $\begin{array}{l}\mathrm{Q} \\
\mathrm{A} \\
\mathrm{W}\end{array}$ & $\begin{array}{l}236 \pm 35 \\
313 \pm 66 \\
403 \pm 115\end{array}$ & 32.6 & $\begin{array}{r}7.62 \pm 0.20 \\
8.81 \pm 0.82 \\
10.38 \pm 1.79\end{array}$ & 15.6 & $\begin{array}{l}129.4 \pm 4.0 \\
142.5 \pm 7.4 \\
162.5 \pm 9.4\end{array}$ & 10.1 \\
\hline 3 & $\begin{array}{l}\mathrm{Q} \\
\mathrm{A}\end{array}$ & $\begin{array}{l}295 \pm 33 \\
302 \pm 25\end{array}$ & 5.4 & $\begin{array}{l}7.55 \pm 0.94 \\
7.40 \pm 0.75\end{array}$ & -2.0 & $\begin{array}{l}144.1 \pm 5.5 \\
150.1 \pm 4.3\end{array}$ & 4.2 \\
\hline 4 & $\begin{array}{l}\mathrm{Q} \\
\mathrm{A} \\
\mathrm{W}\end{array}$ & $\begin{array}{l}353 \pm 50 \\
372 \pm 72 \\
403 \pm 84\end{array}$ & 5.4 & $\begin{array}{l}6.74 \pm 0.29 \\
7.20 \pm 0.74 \\
7.99 \pm 0.53\end{array}$ & 6.8 & $\begin{array}{l}161.7 \pm 7.1 \\
164.2 \pm 11.0 \\
164.0 \pm 4.9\end{array}$ & 1.5 \\
\hline 5 & $\begin{array}{l}\text { Q } \\
\mathrm{A} \\
\mathrm{W}\end{array}$ & $\begin{array}{l}232 \pm 21 \\
241 \pm 38 \\
216 \pm 50\end{array}$ & 3.9 & $\begin{array}{l}9.06 \pm 0.66 \\
9.11 \pm 1.01 \\
9.45 \pm 1.33\end{array}$ & 0.6 & $\begin{array}{l}154.2 \pm 9.1 \\
158.6 \pm 13.2 \\
171.3 \pm 6.2\end{array}$ & 2.9 \\
\hline 6 & $\begin{array}{l}\mathrm{Q} \\
\mathrm{A} \\
\mathrm{W}\end{array}$ & $\begin{array}{l}275 \pm 92 \\
321 \pm 67 \\
477\end{array}$ & 16.7 & $\begin{array}{l}9.20 \pm 1.38 \\
9.90 \pm 1.00 \\
12.5\end{array}$ & 7.6 & $\begin{array}{l}163 \pm 9.6 \\
172 \pm 6.6 \\
187\end{array}$ & 5.5 \\
\hline 7 & $\begin{array}{l}\mathrm{Q} \\
\mathrm{A} \\
\mathrm{W}\end{array}$ & $\begin{array}{l}307 \pm 36 \\
377 \pm 67 \\
465 \pm 57\end{array}$ & 22.8 & $\begin{array}{r}7.70 \pm 39 \\
8.84 \pm 1.04 \\
10.24 \pm 0.52\end{array}$ & 14.8 & $\begin{array}{l}141.4 \pm 3.5 \\
152.6 \pm 7.2 \\
161.9 \pm 5.8\end{array}$ & 7.9 \\
\hline 8 & $\begin{array}{l}\mathrm{Q} \\
\mathrm{A}\end{array}$ & $\begin{array}{l}186 \pm 42 \\
282 \pm 103\end{array}$ & 51.6 & $\begin{array}{l}6.26 \pm 0.80 \\
8.24 \pm 1.11\end{array}$ & 31.6 & $\begin{array}{l}147 \pm 10.3 \\
163 \pm 12.2\end{array}$ & 15.0 \\
\hline 9 & $\begin{array}{l}\mathrm{Q} \\
\mathrm{A} \\
\mathrm{W}\end{array}$ & $\begin{array}{l}312 \pm 20 \\
389 \pm 40\end{array}$ & 24.7 & $\begin{array}{l}8.23 \pm 0.31 \\
8.76 \pm 0.35 \\
9.90 \pm 1.44\end{array}$ & 6.4 & $\begin{array}{l}156 \pm 2.6 \\
159 \pm 3.4\end{array}$ & 1.9 \\
\hline 10 & $\begin{array}{l}\mathrm{Q} \\
\mathrm{A} \\
\mathrm{W}\end{array}$ & $\begin{array}{l}263 \pm 14 \\
282 \pm 42 \\
347 \pm 76\end{array}$ & 0.4 & $\begin{array}{l}8.12 \pm 0.3 \\
8.38 \pm 0.38 \\
9.46 \pm 0.88\end{array}$ & 3.2 & $\begin{array}{l}144 \pm 2.6 \\
150 \pm 3.8 \\
156 \pm 12.9\end{array}$ & 4.2 \\
\hline 11 & $\begin{array}{l}\text { Q } \\
\text { A } \\
\text { W }\end{array}$ & $\begin{array}{l}389 \pm 107 \\
468 \pm 141 \\
591 \pm 278\end{array}$ & $\begin{array}{r}16.9 \\
\text { mean }=18.4\end{array}$ & $\begin{array}{r}10.06 \pm 1.58 \\
11.2 \pm 1.52 \\
11.9 \pm 2.17\end{array}$ & $\begin{array}{r}10.2 \\
\text { mean }=10.3\end{array}$ & $\begin{array}{l}165 \pm 4.2 \\
173 \pm 7.3 \\
190 \pm 20.9\end{array}$ & $\begin{array}{c}3 \\
\text { mean }=6.4\end{array}$ \\
\hline
\end{tabular}

\footnotetext{
'A, active sleep; Q, quiet sleep; W, wakefulness.

${ }^{2}$ Mean \pm S.D.
}

these observations, it is unlikely that the experimental intervention itself accounts for the recorded changes in $\dot{\mathrm{V}}_{\mathrm{I}}, \dot{\mathrm{V}}_{\mathrm{O}_{2}}$, and HR.

We suggest that the postfeeding time trend in $\mathrm{V}_{\mathrm{I}}, \dot{\mathrm{V}}_{\mathrm{O}_{2}}$, and HR seen in low birth infants can best be explained by specific dynamic action or thermic effect of food, i.e., the increase in energy exchange which follows ingestion of food. This increase in heat production reaches a peak approximately $30 \mathrm{~min}$ after feeding, then steadily decreases until the infant is fed again, and in growing infants, produces a 15 to $20 \%$ increase in $\dot{\mathrm{V}}_{\mathrm{O}_{2}}$ (5). The thermic response to food is particularly prominent in growing infants, perhaps because of a high rate of protein synthesis. $\dot{V}_{I}$ and HR increase with increased $\dot{\mathrm{V}}_{\mathrm{O}_{2}}$, and parallel changes in these variables would be presumed to occur concurrently with the thermic effect of food $(4,20)$.

Our studies usually began more than 30 min after a feeding; therefore, data were usually obtained during the time of peak or decreasing thermic effect, i.e., when $\dot{\mathrm{V}}_{\mathrm{O}_{2}}$ was high and beginning to fall. The decrease in energy expenditure which occurs as the thermic effect is abating could thus account for the observed changes in all variables. This effect operates independently of the state of the infant, producing more gradual, but significant, changes in physiology upon which the state-related changes are superimposed. As described above, this trend is apparent within single epochs, but is even more obvious between epochs of the same state at different postprandial intervals.

We conclude from data obtained in our studies as well as from evidence obtained by other investigators that the state of sleep and wakefulness is a major influence on the physiology of low birth weight infants. However, in the design of experiments involving low birth weight infants it is also necessary to account for an additional significant influence on metabolism and respiration which is related to the feeding cycle. Some of the discrepancy in published observations of the respiration, metabolism, and circulation of low birth weight infants might be explained by failure to control for these effects. In this study, comparisons were confined to continuous observations of individual infants. Obviously, the potential effects of differences among infants related to weight, gestational age, postnatal age, growth rate, and other factors must 
Table 3. Slope of regression line of $\dot{V}_{1}, \dot{V}_{O_{2}}$, and HR against time within an epoch of active and quiet sleep

$$
\dot{\mathrm{V}}_{1} \quad \dot{\mathrm{V}}_{\mathrm{O}_{2}} \quad \mathrm{HR}
$$

Patient $\quad(\mathrm{ml} / \mathrm{kg}-\mathrm{min} / \mathrm{hr}) \quad(\mathrm{ml} / \mathrm{kg}-\mathrm{min} / \mathrm{hr}) \quad($ beats $/ \mathrm{min} / \mathrm{hr})$

Quiet sleep

$\begin{array}{cccc}1 & 69 & 2.9 & 0 \\ 1 & -202 & -2.3 & -6.32 \\ 2 & -61 & -0.33 & 6 \\ 4 & -4.8 & -1.64 & -28 \\ 5 & -24.4 & -3.75 & -2 \\ 6 & -42.8 & -0.167 & -13 \\ 6 & -106 & -0.012 & -9.8 \\ 8 & -78.4 & -0.116 & -0.147 \\ 10 & -73.6 & -1.60 & 10.3 \\ 10 & -144 & -2.25 & -25.2 \\ 11 & -310 & -3.6 & -10 \\ 11 & -278 & -5.8 & -33.3\end{array}$

Active sleep

\begin{tabular}{rrrr}
1 & -525 & -1.69 & -29.5 \\
2 & -217 & -3.41 & -35.4 \\
3 & 23 & -2.70 & -15.2 \\
4 & -183 & -1.64 & -24.4 \\
6 & -71 & -1.01 & -18.9 \\
8 & -256 & -0.249 & -56.2 \\
10 & -109 & -1.16 & -22.6 \\
11 & 0 & 1.89 & 3.2 \\
11 & -261 & -3.10 & -7.75 \\
& & & \\
Mean \pm S.D. & $-156 \pm 149$ & $-1.49 \pm 1.94$ & $-15.0 \pm 15.9$ \\
\hline
\end{tabular}

Table 4. Changes in $\dot{V}_{l}, \dot{V}_{\mathrm{O}_{2}}$, and $H R$ between two successive epochs of quiet and active sleep occurring in the same feeding interval

\begin{tabular}{|c|c|c|c|}
\hline \multirow[b]{2}{*}{ Patient } & \multicolumn{3}{|c|}{$\%$ change } \\
\hline & $\frac{\dot{\mathrm{V}}_{\mathrm{L}_{1}}-\dot{\mathrm{V}}_{\mathrm{L}_{2}}}{\dot{\mathrm{V}}_{\mathrm{L}_{1}}} \times 100$ & $\frac{\dot{\mathrm{V}}_{\mathrm{O}_{21}}-\dot{\mathrm{V}}_{\mathrm{O}_{22}}}{\dot{\mathrm{V}}_{\mathrm{O}_{21}}} \times 100$ & $\frac{\mathrm{HR}_{1}-\mathrm{HR}_{2}}{\mathrm{HR}_{1}} \times 100$ \\
\hline \multicolumn{4}{|l|}{ Quiet sleep } \\
\hline 6 & -46.6 & -23 & -9.7 \\
\hline 9 & -37 & -7.3 & -2.6 \\
\hline 10 & -5.9 & -4.7 & +1.8 \\
\hline 11 & -40.0 & -24.6 & -2.2 \\
\hline \multicolumn{4}{|l|}{ Active sleep } \\
\hline 6 & -27.6 & -14.9 & -4.0 \\
\hline 9 & -18.9 & -14.7 & -4.5 \\
\hline 10 & -14.0 & -7.8 & -3.9 \\
\hline 11 & -31.8 & -18.3 & -0.0 \\
\hline
\end{tabular}

also be considered in planning respiratory and metabolic studies of groups of low birth weight infants.

\section{REFERENCES AND NOTES}

1. Anders, T. F.. Ende, R., and Parmelee, A. H.: A Manual of Standardized Terminology Techniques and Criteria for the Scoring of Status of Sleep and Wakefulness in Newborn Infants. (Brain Information Service, UCLA, Los Angeles, CA, 1971).
2. Ashton. R., and Connolly. K.: The relation of respiration rate and heart to sleep states in the human newborn. Dev. Med. Child Neurol., 13: 180 (1971).

3. Bolton, D. P. G.. and Herman, S.: Ventilation and sleep state in the newborn. J Physiol. (Lond.), 240: 67 (1974).

4. Bradfield. R. B.: A technique for determination of usual daily energy expenditure in the field. Am. J. Clin. Nutr., 24: 1148 (1971).

5. Brooke, O. G., Alvear, I, and Arnold. M.: Energy retention, energy expenditure and growth in healthy immature infants. Pediatr. Res., 13: 215 (1979).

6. Bruck. K.: Temperature regulation in the newborn infant. Biol. Neonat., 3: 65 (1961).

7. Brück, K., Parmelee, A. H., and Brück, M.: Neutral temperature range and range of "thermal comfort" in premature infants. Biol. Neonat., 4: 32 (1962).

8. Curzi-Dascalova. L., and Gaudebout, C. A.: Respiratory frequencies of sleeping infants during the first months of life. Abst. Association for Psychophysiological Study of Sleep. 20th Annual Meeting. Unidad de Congressos Centro Medico Nacional. Mexico, D.F. Mexico, 264 (1980).

9. Dreyfus-Brisac, C.: Ontogenesis of sleep in human prematures after 32 weeks of conceptional age. Dev. Psychobiol., 3: 31 (1970).

10. Fahri. L. E., and Rahn, H.: Gas stores in the body and the unsteady state. J. Appl. Physiol., 7: 472 (1955).

11. Hasselbalch, R. A.: Respiration forsag paa Nyfadte Barn, Bibl. Laeger, 8: 219 (1904).

12. Kattwinkel, J.; Nearman, H. S., Fanaroff, A. A.; Katona. P. G., and Klaus, M. $\mathrm{H}$ : : Apnea of prematurity, comparative therapeutic effects of extraneous stimulation and nasal continuous positive airway pressure. J. Pediatr. 86: 588 (1975).

13. Krauss, A. N., Brown, J., Waldman, S., Gottleib, G., and Auld. P. A. M. Pulmonary function following feeding in low birth weight infants. Am. J. Dis. Child., 132: 139 (1978).

14. Lister, G., Hoffman, J. E., and Rudolph, A. M.: Oxygen uptake in infants and children: a simple method for measurement. Pediatrics, 53: 656 (1974).

15. Mestyan. J.. Jara. I., Kekete, M.. and Soltesz, G. Y.: Specific dynamic action in premature kept at and below the neutral temperature. Pediatr. Res., 3: 41 (1969).

16. Parmelee, A. H., Schulte. F. J.. Akiyama, Y., Wenner, W. H., Schultz. M. A., and Stern, E.: Maturation of EEG activity during sleep in premature infants. Electrencephologr. Clin. Neurophysiol., 24: 319 (1968).

17. Prechtl, H. F. R.: The behavioral states of the newborn infant (a review). Brain Res., 76: 185 (1974).

18. Rigatto, H., and Brady, J.: Periodic breathing and apnea in preterm infants. I Evidence for hypoventilation possibly due to central respiratory depression. Pediatrics, 50: 202 (1972).

19. Kalapesi, Z., Leahy, F. N., Cates, D., MacCallum, M., and Rigatto, H.: Evidence for control of tidal volume and frequency being metabolically dependent during REM and N-REM sleep in preterm infants. Pediatr. Res. (Abstract). 13: 536 (1979).

20. Schulze, K., Kairam, R.. Indyk, L.. Wung, J., Sciacca, R., Dell, R., and James, L. S.: The relationship between minute ventilation, oxygen consumption and carbon dioxide production in the newborn. Pediatr. Res. (Abstract), 13: 1289 (1979).

21. Schulze, K., Kairam R., Stefanski. M., Sciacca, R., and James, L. S.: Continuous measurement of minute ventilation and gaseous metabolism of newborn infants. J. Appl. Physiol., 50: 1098 (1981).

22. Scopes, J. W., and Almed, I.: Minimal rates of oxygen consumption in sick and premature infants. Arch. Dis. Child., 41: 407 (1966).

23. Siassi, B., Hodgeman, J. E., Cbal. L.. and Hon. E. M.: Cardiac and respiratory activity in relation to gestation and sleep states in newborn infants. Pediatr. Res., 13: 1163 (1979).

24. Stabell, V., Junge. M.. and Fenner, A.: Metabolic rate and $\mathrm{O}_{2}$ consumption in newborns during different states of vigilance. Biol. Neonate, 31: 27 (1977).

25. Stothers, J. K., and Warner, R. M.: Oxygen consumption and neonatal sleep states. J. Physiol. (Lond.), 278: 435 (1978).

26. Swyer, P.: Heat loss after birth. In: J. D. Sinclair: Temperature Regulation and Energy Metabolism in the Newborn. pp. 91-128 (Grune \& Stratton, New York, 1978).

27. Watanabe, K.. Iwase, K., and Hara. K.: Heart rate variability during sleep and wakefulness in low birth weight infants. Biol. Neonate, 22: 87 (1973).

28. Widdicombe,d J. G.: Reflex control of breathing. Respir. Physiol., 273 (1974).

29. Yu. V. Y. H., and Rolfe, P.: Effect of feeding on ventilation and respiratory mechanics in newborn infants. Arch. Dis. Child, 51: 310 (1976)

30. Requests for reprints should be addressed to: Dr. Karl Schulze, College of Physicians and Surgeons of Columbia University, 3975 Broadway, New York, NY 10032 (USA).

31. Supported by the United States Public Health Service SCOR Grant HL 14218.

32. Received for publication August 28, 1980.

33. Accepted for publication December 12, 1980. 